\title{
Les Représentations De La Beauté Féminine: Une Lecture Des Fondements Socioculturels Du Teint Clair Chez Les Bété De Côte d'Ivoire
}

\begin{abstract}
Yoro Blé Marcel
Enseignant-chercheur à l'Institut des Sciences Anthropologiques de Développement (ISAD), UFR Sciences de l'Homme et de la Société, Université Félix Houphouet-Boigny, Abidjan-Cocody, Côte d'Ivoire
\end{abstract}

doi: 10.19044/esj.2017.v13n23p39 URL:http://dx.doi.org/10.19044/esj.2017.v13n23p39

\begin{abstract}
This study proposes an analysis of the representation of the female beauty whose clear complexion is one of the illustrations in the bété. In Côte d'Ivoire, the bété shows that there was a development of the beauty through the worship of the bagnon. However, in the representation of the male beauty through the bagnon, there is no fixed color complexion. It is not the same for the beauty of the woman whose clear complexion is of significant importance. This is why this study proposes the canons of the female beauty and to question the sociocultural bases which specifically underlie the preference of the clear complexion to the black complexion of the bété woman. However, this is in a bid to understand the cultural anchoring of the female depigmentation in bété country. From a field survey of people of all social categories, the study shows that the preference of the clear complexion is explained by the fact that it would carry virtues of rejuvenation and longevity for the man. In other words, the beauty of the woman aims at developing the man by rejuvenating him and by lengthening his life expectancy. These imaginations results to the depigmentation of the young girls and women bété in their search for beauty. This they do via the clear complexion to make them more attractive to men.
\end{abstract}

Keywords: Female beauty, depigmentation, representation, tint clearly, bête.

\section{Resume}

Cette étude propose une analyse des représentations de la beauté féminine dont le teint clair est l'une des illustrations chez les bété. En Côte d'Ivoire, c'est chez les bété qu'il y a eu une mise en valeur de la beauté à travers le culte du bagnon. Mais, alors que dans les représentations de la beauté masculine à travers le bagnon, il n'y a aucune fixation sur la couleur 
du teint, il n'en est pas de même pour la beauté de la femme, pour laquelle le teint clair est d'une importance capitale. C'est pourquoi cette étude se propose de s'interroger sur les canons de la beauté féminine et de questionner les fondements socioculturels qui sous-tendent la préférence du teint clair au teint noir spécifiquement chez la femme bété, en vue de comprendre l'ancrage culturel de la dépigmentation féminine en pays bété. L'étude montre, à partir d'une enquête de terrain auprès de personnes de toutes catégories sociales, que la préférence du teint clair s'explique par le fait qu'il serait porteur de vertus de rajeunissement et de longévité pour l'homme. En d'autres termes, la beauté de la femme vise à valoriser l'homme en le rajeunissant et en rallongeant son espérance de vie. Ce sont donc ces imaginaires qui sous-tendent la dépigmentation des jeunes filles et femmes bété dans leur quête de la beauté, via le teint clair, pour plaire aux hommes.

Mots-clés: Beauté féminine, dépigmentation, représentation, teint clair, bété

\section{Introduction}

Dans toutes les sociétés, la beauté a toujours fait objet de constructions sociales. A chaque société donc, ses canons ou critères de beauté. Il n'y a donc pas de beauté universelle malgré les tentatives d'universalisation de la beauté féminine à travers les concours de Miss dans le monde, qui tentent d'imposer les canons de beauté propres aux sociétés occidentales.

De nos jours, la recherche de la beauté physique féminine version européenne, s'accompagne chez les africaines notamment, par le désir, si non la recherche du teint clair, qui est l'un des éléments principaux de la beauté contemporaine. En effet, on a l'impression que des femmes africaines d'aujourd'hui veulent presque toutes être blondes, claires ou simplement blanches. D'où la prolifération des pratiques de dépigmentation dans un contexte où fleurissent les produits cosmétiques dépigmentant. Toutes ces pratiques traduisent la nouvelle dynamique de la beauté telle qu'appréhendée par les nouvelles générations. En effet, dans chaque société, le corps se définit comme un objet d'apparence. La façon dont il est mis en valeur donne des indications sur les sociétés. Cela est encore vrai pour les sociétés africaines. En effet, dans la plupart de celles-ci, il n'y a pas de rupture entre le corps et l'environnement social. Parce que le corps relie l'individu à la société, les soins apportés au corps sont d'une grande importance. Il faut présenter un corps acceptable dans la société où l'on vit ou par son entourage immédiat. L'esthétique du corps trouve toute son importance dans cette perspective. C'est ainsi que les critères de beauté du corps varient selon les sociétés. La beauté est donc un problème d'identité 
culturelle. Comme le souligne Detrez (2002:16), quelle que soit la perspective, la représentation du corps dans une société reflète ses valeurs culturelles.

En Côte d'Ivoire, quand on parle de la beauté, l'on ne peut s'empêcher de penser au peuple bété. En effet, c'est ce groupe ethnique qui a fait de la beauté, une institution dénommée bagnon, littéralement, « bel homme »:

«Il existe dans la société traditionnelle des Bété en Côte d'Ivoire, une institution relative à la beauté : l'institution du bagnon. Il s'agit d'un véritable culte rendu à la beauté et organisé autour d'un homme qui incarne aux yeux de la communauté villageoise, les attributs de la beauté physique » (Wondji, 1986 :43).

La beauté chez les bété renvoie donc à un problème de genre, c'est-àdire une construction «sociale, historique, sociologique et culturelle de ce qu'est (ou devrait être) une femme ou un homme, le féminin ou le masculin » (Borghino, 2003), car le bagnon est une institution exclusivement masculine. La beauté de la femme n'est pas exhiber comme celle de l'homme. Pour expliquer cette exclusion de la femme, deux propositions théoriques ont été avancées par certains auteurs: l'une qu'on pourrait qualifier de naturaliste, et l'autre, culturelle. Selon la première, «... la femme plaît naturellement. On n'a donc pas besoin pour elle de mesures spéciales » (Bony, 1967:9) ; pour la seconde :

«Du point de vue de l'idéologie, la femme est non-valeur dans le cadre de la suprématie masculine qui accorde au sexe mâle tous les attributs de la positivité : force, intelligence, bien; être subordonné, la femme est minorisée à l'instar de tous les cadets sociaux (fils, fille, esclave, etc.) : c'est la pauvre femme (wonon gbagbaflè) » (Wondji, 1986:54).

C'est dans la perspective de cette deuxième théorie que s'inscrit notre travail qui va d'abord, à partir des enquêtes sur le terrain, définir ce qu'est la beauté et son importance socioculturelle chez les bété, ensuite interroger les canons de beauté de la femme chez ce peuple et enfin questionner les fondements socioculturels qui sous-tendent la préférence du teint clair au teint noir chez la femme, en vue de comprendre l'ancrage culturel de la dépigmentation féminine chez les jeunes filles et femmes bété de nos jours.

Au plan méthodologique, notre étude s'est basée sur des observations personnelles, de plusieurs entretiens directs et semi-directifs auprès de plusieurs personnes dans les villages de Gazahio, Beugréhoa et Bagasséhoa, dans la sous-préfecture de Dignago (dans le département de Gagnoa, au centre-ouest de la Côte d'Ivoire), du 15 octobre au 12 novembre 2015. L'étude a été menée auprès de 22 personnes composées d'adultes hommes et femmes, ainsi que de jeunes hommes et filles dont certains se dépigmentent la peau à la recherche du teint clair. L'âge des enquêtés varie de 18 à 82 ans. 


\section{Définition de la beauté et son importance socioculturelle}

En Côte d'Ivoire, les bété appartiennent au grand groupe socioculturel Kru situé au Centre-Ouest du pays. Et parmi les peuples Kru, c'est chez eux qu'il a existé un culte de la beauté, le culte du bagnon. En bété, $b a$ est un adjectif utilisé pour qualifier toute personne ou toute chose présentant une valeur esthétique et qui signifie "beau" ou "belle". Le bel homme se dit bagnon, gnon étant un substantif signifiant homme, dans le sens d'être humain mâle par opposition à femme, wonon. Cependant $b a$ peut être associé à la femme, on parle alors de bawonon, une belle femme. La création de cette institution dédiée à la beauté masculine témoigne de l'importance sociale et culturelle que représentait la beauté dans cette société. Le bel homme est un yilimenènegnon (de yili = œil, nenè = beau, bon, et gnon $=$ homme) c'est-à-dire « celui qui est beau à voir ». De même, la belle femme est une yilimenènewonnon. Mais tout bel homme ou belle femme n'induit pas nécessairement un bagnon ou une bawonnon. Il/elle doit être un nékpéha nékpê (un homme bon ou une belle âme) ou wonnonha wonnon (une bonne femme ou une belle âme). En effet, pour les bété, le corps et l'esprit font un, donc celui/celle qui est belle physiquement doit aussi l'être moralement. Par exemple, la belle femme bété ne doit pas être srowonon, c'est-à-dire "une femme volage». Elle doit être une femme généreuse, fidèle, féconde. Dans la mentalité des bété, comme dans plusieurs groupes ethniques ivoiriens (Memel-Fotê, sous la dir. De, 1998), l'homme, nyikpè, est composé de $k u$ ou kpekpa (corps) : la partie extérieure et visible de l'homme. Il s'agit du corps qui désigne la personne humaine dans son intégralité et dans son aspect extérieur. Comme le disait à juste titre Le Breton (1990:7) : «Le corps est un thème particulièrement propice pour l'analyse anthropologique puisqu'il appartient de plein droit à la souche identitaire de l'homme. Sans le corps qui lui donne un visage, l'homme ne serait pas ». L'existence de l'homme est donc corporelle. Les bété assignent à l'esthétique de ce corps visible (Yoro, 2006), une importance capitale, d'où l'institution bagnon, qui est la célébration de cette beauté plastique. Comme le dit si bien Wondji (op.cit.:43) «La beauté du bagnon imprègne le corps humain tout entier, depuis la tête jusqu'aux pieds». Le bagnon est donc un concept à travers lequel s'exprime une certaine vision du beau chez les bété. Les éléments de la beauté physique du bagnon sont selon Wondji (op.cit.:47) :

"La régularité et la pureté des lignes du modèle, l'équilibre des formes, l'harmonie plastique d'ensemble du corps humain... L'harmonie des proportions et non l'ampleur des formes, tel est le canon de base de la beauté plastique des bété perçue à travers l'esthétique du bagnon ".

Et pour mettre en exergue cette beauté du corps, les bété se réfèrent souvent aux exemples de la nature animale et végétale pour former les 
concepts nécessaires à celle-ci, tout comme ils le font aussi pour leur réflexion sur le monde et la société. Aussi, l'idée du beau est-elle souvent rendue à partir de certaines qualités physiques des plantes et des animaux. C'est ainsi que, comme le souligne Wondji (op.cit.:45), parmi les arbres, ils se réfèrent au zizé (le sipo), c'est-à-dire l'entandrophragunautile de la famille des méliacées, qui a un tronc svelte, lisse et joliment coloré. Il est considéré comme un modèle de beauté et donne son nom aux hommes et femmes dont le corps représente les mêmes qualités. Le bel homme/belle femme est donc ziza au beau corps. Les comparaisons tirées du monde animal sont aussi légion. Glé (le cercopithèque) est un singe au charme chatoyant à cause de son pelage richement décoré, de son corps assez bien proportionné. Il est considéré comme un bel animal, le plus beau des singes, mais aussi le plus intelligent. D'un homme/une femme qui a une belle peau, un corps propre et bien entretenu, on dit qu'il/elle est gléku (il/elle a le corps de glé), classé parmi les plus beaux des animaux. De même, ils se réfèrent aussi à la panthère $(g b i)$ jugée belle à cause de sa robe joliment tachetée, et de ses gestes élégants et gracieux. On parle ici de gbiku (corps de panthère). Il en est de même de la peau de l'antilope et du léopard.

Il ressort de ces comparaisons que la qualité de la peau et le mouvement du corps, entrent dans la composition du beau en ajoutant à la structure physique des éléments aussi importants que l'élégance ou la sveltesse, le charme des couleurs, la prestance et la grâce. Lorsque ces qualités se dégagent des corps, elles contribuent à l'accroissement de la beauté et sont de ce fait des attributs de celle-ci. Tel est le canon de base de la beauté plastique des bété perçue à travers le bagnon/bawonon. La beauté demeure donc une question d'agencement harmonieux du corps, une harmonie plastique de l'ensemble du corps humain. Mais, contrairement au bagnon qui est exposé, exhibé, ce que le bété appelle sibhé sibhé, la bawonon elle, n'est pas exhiber. Ceci s'explique entre autres par la primauté de la fonction de reproduction de la femme, indépendamment de ses critères de beauté. Il n'en demeure pas moins vrai que la beauté féminine répond à des critères spécifiques qui lui sont propres.

\section{Les critères de la beauté féminine}

En pays bété, il n'existe pas de culte de la beauté féminine comme pour l'homme, mais il existe bel et bien des critères de beauté de la femme. Même si la belle femme en bété n'est pas perçue comme un objet d'art qu'il faut exhiber, on exige de la bawonon des qualités esthétiques physiques et morales. Elle doit être agréable à voir, à contempler, à dévorer du regard : «bawonon digbiguemo wayèrènon », ce qui veut dire que la belle femme, on la regarde sur le dos des mortiers. Cela signifie qu'elle est tellement belle, et que tout le monde voulant la regarder, il n'y a quasiment plus de place pour 
se frayer un chemin, donc pour la voir, il faut renverser un mortier et monter là-dessus pour pouvoir la voir. Cette symbolique traduit la popularité de la belle femme dans la société bété. Cela signifie qu'elle ne passe pas inaperçue et quiconque la croise ne manque pas de la suivre du regard. C'est pourquoi plusieurs anthroponymes féminins font référence à la beauté pour celles présumées belles. Nous avons entre autres, gougouhehisi (de gougouhehi = maîs et si = brûler): « belle femme qu'on se plaît à contempler au point de laisser son maïs brûler sur le feu »; ônané : belle, gentille, généreuse »; yila (yeux): « une femme qu'on contemple, qui attire les yeux », ou yirigané ( de yiri = yeux et gané $=$ suit): « celle que l'œil suit, ou celle qui ne passe pas inaperçue »; dactchèlé (de da = mère et tchèlé = dessiner): « belle femme dessinée par sa mère $»$; etc.

Ces noms ne sont pas donner au hasard, mais tiennent compte des canons de beauté de celles qui les portent. Dès la naissance déjà, les bété détectent ces indices de beauté chez l'enfant. En effet, les yeux sont les organes d'accès aux mouvements plastiques, c'est pourquoi on dit en bété yirimo onénéè, littéralement, il/elle est belle à la vue. Le regard porte sur le corps tout entier de la tête aux pieds.

Selon les informateurs, les canons de beauté de la femme partent de la tête aux pieds en commençant par la stature : la belle femme doit être grande. Même s'ils ne disent pas exactement la taille qui convient, on peut tout de même penser à environ $1 \mathrm{~m} 75$ au moins à travers 1'expression wonon a vavahi c'est-à-dire « une femme grande, élancée » car cela implique l'élégance : "la belle femme doit tout de suite attirer l'attention. Donc elle doit avoir un aspect qui sort de l'ordinaire, comme par exemple une taille imposante ». La belle femme ne saurait donc être une naine ; elle doit avoir un visage ovale avec des sourcils noirs bien dessinés. Le visage beau l'est de face comme de profil. Avoir de long cheveux comme ceux d'une femme blanche ; le cou doit être plissé et dégagé du tronc ; les yeux ne doivent pas être petits et cachés dans les orbites. Ils ne doivent pas être non plus exagérément gros. Ils doivent être visibles; le nez doit être droit «comme pour les blancs» et en harmonie avec le visage. "Quand on regarde le visage de la belle femme, on ne doit pas voir tout de suite son nez du fait de sa disproportion, de sa grosseur comme celui du kpè, cet oiseaux au gros nez, ou de sa petitesse (comme celui du singe), mais tout doit être harmonieux $»$; les gencives noires avec des dents très blanches assorties d'un écart central ou d'une brèche. La gencive doit être naturellement colorée pour laisser transparaître la blancheur des dents. L'écart central rehausse la beauté du sourire et met plus en exergue la blancheur des dents, ce qui rejaillit sur le physique de la belle femme ; les seins développés affirment la féminité de la femme, mais c'est aussi selon les informateurs hommes, un signe de fertilité ; la belle femme doit être une femme callipyge, c'est-à-dire 
aux fesses fortement développées, rebondies et dégagées du tronc; les jambes bien galbées et droites avec de jolis mollets, différents des mollets têtus ou de coq. Les jambes d'éléphant ne sont pas admises. Elle ne doit pas avoir non plus des jambes croisées ou arquées ou en $\mathrm{X}$, mais des jambes droites de dos comme de face, et en parfaite harmonie avec ses deux paires de fesses. "Ses fesses doivent danser à chacun de ses pas traduisant ainsi son élégance, sa grâce et sa beauté »; Les orteils et les doigts doivent être fins, longs et droits.

Comme on le voit, les critères de la beauté féminine sont très sélectifs et semblent être plutôt de l'ordre de l'utopie. En d'autres termes, la belle femme au sens des bété est une perle rare. Ceci explique sans doute pourquoi les bété se sont gardés de célébrer la beauté féminine contrairement à la beauté masculine à travers le bagnon. En effet, ce qui primait et prime encore pour la femme, c'est sa fonction de reproduction qui n'est nullement mise en cause par sa beauté ou sa laideur.

Cependant, en ce qui concerne la couleur du teint de la femme, il y a une divergence de vues avec un penchant pour le teint clair. La belle femme doit avoir un teint clair. Quels sont les fondements socioculturels de cette assertion?

\section{Les fondements socioculturels du teint clair}

Dans l'imaginaire traditionnelle bété, la beauté de la femme n'a de valeur que par rapport à l'homme. La femme doit être belle pour l'homme. Les propos des informatrices ne disent pas autre chose : "Je me fais belle pour avoir un mari. Les hommes aiment les femmes claires donc il faut faire avec si on veut avoir un mari ». En effet, selon l'idéologie masculine bété, la peau claire est la référence de la beauté. Elle permet de se faire remarquer, admirer. C'est donc un critère social de popularité, de célébrité. Elle est préférée par les hommes car indicatrice d'une bonne santé, d'une aisance sociale et participe à la respectabilité. Des expressions métaphoriques traduisent cette préférence : wonon zrô luwili né gô : "le mari d'une femme claire ne vieillit jamais ». Ainsi donc, la peau claire rajeunit la femme. En d'autres termes, la femme claire paraît toujours jeune du fait du rayonnement de son teint. Or la jeunesse ou l'éclat de la femme étant présumé rejaillir sur celui de son mari, celui-ci reste aussi jeune quel que soit son âge. Le teint clair se présente donc à leurs yeux, comme un gage de jeunesse « éternelle ». C'est aussi un indicateur de bonne santé et même de virilité de l'homme, car on pense qu'il faut être bien portant pour être l'époux d'une jeune femme. C'est aussi un indicateur d'aisance sociale parce qu'on pense que le teint clair nécessite un entretien qui a un coût financier que le mari doit être capable de supporter. Cependant le teint clair dont il s'agit dans ces représentations selon nos informateurs hommes, est bien le teint naturel et 
non le teint artificiel consécutif au blanchiment de la peau. Mais cette idéologie du rajeunissement incite les jeunes filles d'aujourd'hui, mais aussi des femmes mariées ou célibataires, à s'adonner à la pratique du blanchiment de la peau: "c'est mon mari lui-même qui paye mes produits d'éclaircissement ». Selon la seconde expression métaphorique : wonon zrô luwili né kuhu paha: «le mari d'une femme claire ne meurt pas tôt ». Ici, c'est au plan culturel que prend ancrage cette vision des choses. En effet, c'est la pratique du lévirat qui sous-tendrait cela : " A ta mort c'est ton frère qui va prendre ta femme. Quel homme accepterait de mourir tôt pour laisser sa belle femme dans les bras de son frère ». Partant de ce principe, le mari d'une femme claire prend soin de sa santé de peur de mourir prématurément et laisser sa belle femme claire dans les bras de son frère. C'est pourquoi les informateurs pensent que la femme claire prolonge la vie de son mari. Par cette renaissance sociale, cette vision de la beauté féminine participe, pour parler comme Ndiaye (2008), au phénomène du « colorisme » qui hiérarchise insidieusement la société africaine en fonction de la couleur de la peau. En d'autres termes, la dépigmentation des femmes bété d'aujourd'hui prend ancrage dans ces imaginaires qui font de la peau claire de la femme, un « produit de rajeunissement » pour elle-même et pour son mari, mais aussi un « remède » pour relever l'espérance de vie de l'homme. Comme le dit si bien Emeriau (2009), l'éclaircissement de la peau n'est pas uniquement une pratique culturelle, mais un fait bio-culturel dans lequel s'inscrit une logique imaginaire et symbolique propre à la population qui s'éclaircit la peau. Dans cette logique, cet acte signifie faire « peau neuve », enlever l'ancienne peau porteuse de stigmates liés à la peau «noire », se débarrasser d'une peau encombrante pour laisser place à une nouvelle peau, déchargée de son poids symbolique et qui révèlerait la beauté « naturelle » de l'individu.

De l'avis des informateurs notamment adultes hommes, le teint clair rehausse la beauté de la femme, et valorise son mari, mais une belle femme doit aussi être fertile : «Ce qui compte pour une femme, c'est sa capacité de reproduction. Cette fonction prime sur tout $»$. En effet, par le passé, la famille bété se caractérisait par la quête permanente du nombre (zunon), le nombre ou la quantité étant puissance, prestige, richesse et sécurité. Or c'est la femme, par sa fonction de reproductrice qui pouvait permettre à la société d'atteindre cet objectif. Et c'est ce qui sous-tend la stratégie matrimoniale (Dozon, 1985:88) selon laquelle: il faut des épouses, beaucoup d'épouses non seulement pour la perpétuation du lignage, mais aussi l'agrandissement de la famille par la règle du mariage exogamique. Et c'est cette quête du nombre qui justifie entre antres l'institution bagnon. En effet, «...grâce à son action d'émulation bénéfique, le banion contribue puissamment au revigorement $d u$ potentiel démographique de son groupe» (Holas, 
1980:334). Et pour cela l'exogamie en ligne paternelle était poussée très loin :

"L'institution du bagnon, en valorisant l'homme, permet d'attirer dans le groupe concerné un plus grand nombre de femmes d'autres groupes. Cela est d'autant plus nécessaire que le mariage obéit à des règles rigoureuses d'exogamie : l'exogamie en ligne paternel est poussée très loin. Un homme ne peut épouser les filles de même ancêtre que lui, et la femme non plus ne peut épouser les descendants de cet ancêtre unique. L'homme donc, par sa beauté, par son pouvoir de séduction, par la publicité qui s'instaure autour de sa personne, attirera dans son clan des jeunes filles et des femmes qui accroîtront grâce aux effets de mariage la puissance du groupe... » (Bony, op.cit.:9).

De façon explicite, la stratégie exogamique empêche ego de prendre épouse dans le patrilignage de sa mère, dans celui de la mère de sa mère, dans celui de la mère de son père, enfin dans le gligbë (patrilignage) de la mère du père de son père (Dozon, op.cit.:108). Or justement, la fonction reproductrice de la femme n'est pas liée à sa beauté. Voilà pourquoi les bété n'ont pas mis en place une institution de célébration de la beauté féminine, privilégiant ainsi son rôle de mère. En effet, bien que la femme africaine cumule souvent plusieurs rôles, comme le souligne Oppong (1988), c'est celui de mère qui est privilégié et valorisé par la communauté. Le prestige de la maternité dans cette société étant intimement lié à la vitalité et à la perpétuation du lignage, la femme n'existera socialement que lorsqu'elle aura donné des enfants à sa belle famille (Vinel, 2005). Mais le contexte socioculturel et économique d'aujourd'hui tend à reléguer au second plan, la beauté masculine comme moyen de captation de la gente féminine. Du coup, l'institution bété dédiée à la beauté, notamment à la beauté masculine se trouve mise en cause dans sa fonction socioculturelle. Les canons de beauté de la femme se trouvent donc être mis en avant. Parmi ceux-ci, le teint clair dont on retrouve l'apologie dans la littérature gnomique (contes, légendes, mythes, dictons, proverbes, etc.) des bété, se présente comme une panacée pour les jeunes filles d'avoir un mari, dans le cadre d'une transmission intergénérationnelle (Yoro, Ehui \& Ekra, 2016) de l'idéologie de la beauté féminine chez les bété. En effet.

«La société bété est une société de l'oralité : cela veut dire que la majeure partie de la communication sociale a pour véhicule principal la parole qui constitue ainsi le moyen privilégié et la condition de transmission et de régénération de la culture... » (Dédy, 1986:27).

C'est ainsi que la chanson populaire à l'occasion des funérailles ou de tout autre rassemblement, constitue un moyen privilégié pour véhiculer les valeurs de la société, y compris toute la philosophie de la beauté féminine. Les comportements des jeunes filles s'inspirent ainsi de ces 
valeurs et connaissances véhiculées à ces occasions. Et c'est dans ce sens que la dépigmentation des jeunes filles prend ancrage dans les imaginaires bété de la beauté féminine accordant un intérêt particulier au teint clair.

\section{Conclusion}

En guise de conclusion, on peut retenir que les bété, bien qu'ayant célébré la beauté masculine à travers l'institution bagnon, ont aussi défini des canons de beauté de la femme. Même si, concernant la femme, sa fonction de reproduction a toujours prévalu, et en quelque sorte occulté les critères de la beauté féminine, on retient que la belle femme l'est autant au plan physique que moral. Parmi ses canons de beauté physique, le teint clair occupe une place de choix car porteur selon l'imaginaire bété, de vertus de rajeunissement et de longévité pour la femme, mais surtout pour l'homme. A travers ces représentations, on perçoit une suprématie du masculin sur le féminin, ce que Héritier (1996) appelle «La valence différentielle des sexes », car la beauté de la femme vise avant tout à valoriser l'homme. On peut aussi dire avec Godelier (1982) que l'usage du corps des femmes est une forme de domination masculine, même si Bourdieu (1998) la qualifie de domination masculine consentie. En effet pour plaire aux hommes qui désir le teint clair, les femmes se dépigmentent la peau mettant ainsi en péril leur propre santé. L'esthétique se présente donc comme « une science qui traite du beau et du sentiment que ce beau fait naître en nous » (Bastide, 1977). Toute chose qui sous-tend les pratiques de dépigmentation, surtout des jeunes filles désireuses de se faire belles pour plaire aux hommes. Ces imaginaires mettent ainsi en péril la santé des filles et femmes candidates à la dépigmentation volontaire, car les exposant à des maladies dermatologiques comme les irritations de la peau, les Eczéma, l'Acné, etc., ainsi que l'ont montré les travaux de Mahé A., Kéita S., et Bobin P. (2004) réalisés à Bamako au Mali. L'éclaircissement de la peau est favorisé aujourd'hui, en dehors des constructions socioculturelles de la beauté, par la prolifération des produits éclaircissants et est devenu un phénomène de mode, mais surtout un phénomène transculturel : «...les hommes préfèrent les claires, là aussi quelle que soit la population concernée...D'après des données recueillies systématiquement dans les Human Relations Area Files (catégorie 832: "stimulation sexuelle, idéaux de la beauté érotique, attraction sexuelle »), il y aurait une préférence transculturelle écrasante pour une peau plus claire, avec asymétrie sexuelle caractérisée, la préférence étant édictée par les hommes évaluant les femmes, et jamais l'inverse, cela dans les limites du spectre local de variation chromatique... » (Bonniol, 1995:185-204). Face à ce phénomène, il y a lieu de contrôler davantage ou de règlementer le marché des produits cosmétiques dans les pays africains, et d'encourager les recherches pluridisciplinaires sur la 
question, pour apporter des solutions durables au problème de la transformation du corps.

\section{References:}

1. Bastide, R. (1977). Art et Société, Paris, Payot.

2. Bonniol, J-L. (1995). Beauté et couleur de la peau, In Communications, 60, p. 185-204, http://WWW.persee.fr/web/revues/home/prescript/article/comm_058 8_8018_1995_num_60_1_1918.

3. Bony, J. (1967). «Une institution relative à la beauté dans la société bété : le Bagnon », In bulletin de liaison des Institut d'Ethnosociologie et de Géographie Tropicale, N02.

4. Borghino, B. (2003). Genre et sexe, quelques éclaircissements : www.genreenaction.net/spip.php?article3705.

5. Bourdieu, P. (1998). La domination masculine, Le Seuil.

6. Dedy, S. (1986). Vers une définition de l'art chez les Bété : le mythe de Slèlè, In, La chanson populaire en Côte d'Ivoire, Essai sur l'art de Gabriel Srolou, Sous la dir. De Ch. Wondji Avec la collaboration de B. Kotchy, F. Dédy Séri, A. Kouakou et A. Tapé Gozé, Paris, Présence Africaine, p. 27-41.

7. Detrez, C. (2002). La construction sociale du corps, Paris, Editions du Seuil.

8. Dozon, J-P. (1985). La société bété : histoire d'une ethnie de Côte d'Ivoire, Paris, Karthala.

9. Emeriau, C. (2009). S'éclaircir pour faire « peau neuve »? Corps 2/2009 (7), p111-116. URL:Www.caim.info/revue-corps-dilecta2009-2.page-111.htm.DOI :10.3917/corps.007.0111

10. GODELIER, M. (1982). La production des grands hommes. Pouvoir et domination masculine chez les Baruya de Nouvelle-Guinée, Fayard.

11. Heritier, F. (1996). Masculin/féminin. La pensée de la différence, Odile Jacob.

12. Holas, B. (1980). Traditions Krou, Paris, PUF.

13. Le Breton, D. (1990). Anthropologie du corps et modernité, Paris, PUF.

14. Mahe, A., Keita, S., \& Bobin, P. (1994). Complications dermatologiques de l'utilisation cosmétique des produits dépigmentants à Bamako (Mali), Ann Dornatol Venereol, 121:142-6.

15. Memel-Fotê, H. (Sous la dir de) (1998). Les représentations de la santé et de la maladie chez les ivoiriens, Paris, L'Harmattan.

16. Ndiaye, P. (2008). La condition noire, Essai sur une minorité française, Paris, Calmann-Lévy. 
17. Oppong, C. (1988). «Les femmes africaines : des épouses des mères et des travailleuses ", In Population et société en Afrique au sud du sahara, sous la dir. De Dominique Tabutin, p.321-335, Paris, L'Harmattan.

18. Vinel, V. (2005). Des femmes et des lignages, Ethnologie des relations féminines au Burkina Faso (Moose, Sikoomse), Paris, L'Harmattan, 289p.

19. WondjI, C. (1986). Le bagnon et l'art, In, La chanson populaire en Côte d'Ivoire, Essai sur l'art de Gabriel Srolou, Sous la dir. De Ch. Wondji Avec la collaboration de B. Kotchy, F. Dédy Séri, A. Kouakou et A. Tapé Gozé, Paris, Présence Africaine, p. 42-83.

20. Yoro, B.M. (2006). Les représentations de la maladie et du corps chez les Bété de Côte d'Ivoire, Nyansa-pô, Revue Africaine d'Anthropologie, N0.4, Abidjan, EDUCU, p. 79-90.

21. Yoro, B.M., Ehui, P.A., \& Ekra, J-T. (2016). La survivance des pratiques médicinales ancestrales chez les femmes enceintes à Abidjan (Côte d'Ivoire), Revue Internationale d'Ethnographie, N06, p. 124-134. 